\title{
ON INVARIANT SPEED OF LIGHT
}

\section{S. ORLOV ${ }^{1 *}$}

*lPetrozavodsk State University, Petrozavodsk, 185001, Russia

*Corresponding Author: -

\begin{abstract}
: -
A new physical model of the appearance of light, properties, and its trajectory. Explains the paradox of invariance and isotropy of light based on the laws of classical physics.
\end{abstract}

Keywords: Theory of vortex gravitation, cosmology, cosmogony. Aerodynamics. Invariance speed of light.

\section{(c) (\$) (1)}




\section{INTRODUCTION}

For many years, scientists - physicists have tried to measure the speed of light. Galileo - in the seventeenth century. Early experiment to measure the speed of light was held Ole Roemer, a Danish physicist, in 1676. Another, more accurate way to measure the speed of light is made in Europe Hippolyte Fizi in 1849. Francois Arago determines the speed of light in 1838 by a rotating mirror. In 1862, on the production of the speed of light worked Leon Foucault. In 1926, a result of years of efforts Albert Michelson [1] has had some very high accuracy of the speed of light:

$$
c=299796000 \pm 4000 \mathrm{~m} / \mathrm{s}
$$

The main and paradoxical conclusion in the study of light was his invariance. In other words, the speed of light is the same in all inertial frames of reference is independent of the velocity of the observer and emitter. This surprising fact for the classical physics of his time for the first time proved the Michelson experiment: the independence of the speed of light on the direction (isotropic) and the orbital motion of the Earth around the sun. In the future, this paradox was confirmed by astronomers. In particular, Willem de Sitter in monitoring the spectral binaries found that the speed of light flux from removing the stars and approaches are constant speed of light (c) and equal to each other. That is, they do not depend on the rate of the star (light sources). [2] From the point of view of classical physics this paradox so far can't be explained. Therefore, on the basis of the unknown properties of light in 1905, Einstein proposed the special theory of relativity (STR) in the paper "On the Electrodynamics of Moving Bodies" [3]. SRT conclusions were as paradoxical as the invariance of light. On the basis of the SRT can assert that the events simultaneous in one inertial reference frame will not be simultaneous in another, if these frames of reference are moving relative to each other.

In this article we propose to explain the origin, properties and the invariance of light, in full compliance with the classical laws of physics using the theory of vortex gravitation, cosmology and cosmogony. [4]

\section{LUMINIFEROUS ETHER}

In the theory of vortex gravitation, cosmology and cosmogony all celestial bodies (matter) are essential vortices. The values of the bodies (system of bodies)) and the corresponding vortices may vary to an infinite value. The biggest ethereal vortex that people can watch this universal whirlwind, the smallest - atomic.

The orbital speed of ether in each vortex increases towards the center of an inverse square law. Change of orbital velocity is inversely proportional to the pressure change in the air. Pressure gradient force creates vortex gravitation. This pattern works equally essential in a whirlwind of various volumes. $\mathrm{v}(\mathrm{r})^{2}$

In theory, the vortex gravitation [4] obtained an equation to determine the gravitational force in the etheric vortex:

$$
\mathrm{F}_{\mathrm{II}}=\mathrm{V} \cdot \rho \cdot \frac{\mathrm{v}(\mathrm{r})^{2}}{\mathrm{r}}(1) \text {, Depending on the next }
$$

$V \sim \frac{1}{\sqrt{r}} \quad$ where

$\mathbf{V}$ - volume of the nucleons in the body,

which is in torsion $\rho=8.85 \times 10-12 \mathrm{~kg} / \mathrm{m} 3$ - the density of ether [5] $\boldsymbol{v}(\boldsymbol{r})$ - velocity of ether in the orbit $\mathrm{r} \boldsymbol{r}$ - the radius of the orbit are risen ether vortex

The same relationship between the force of gravity and orbital velocity exists in atomic torsion (vortex).

Each atomic vortex ether rotation occurs continuously around the core with a radius $\mathrm{r}$, which creates the effect of an atomic attraction. For this scheme, gravity can explain the origin of the interatomic forces of attraction. In theory, the vortex gravitation made the condition that the ether penetrates all matter and the body other than atomic nuclei (nucleons). Maximum speed ether several orders of magnitude greater than the speed of light. The rotational speed and the pressure gradient of ether, and the attractive force Fn on the surface atoms reaches a maximum value. Make a guess:

The assumption № 1. Under the action of attractive forces on the surface of the atom or on surface of nucleus of the atom or ether stream is converted into an electromagnetic flux including a light. The light has mass. Gravity act on the light. When converting the ether is the emergence into the light particles (photons) with a mass greater than the mass of the particles of the ether - Amer. Increasing the mass of the particles, according to the law of conservation of angular momentum of rotation, must be accompanied by a decrease inversely proportional to the orbital speed of light. Thus, the light gets its velocity - c.

When orbital revolution of the magnetic flux (light) around the atom to it are two forces: the force of the vortex atomic attraction and centrifugal force. For uniform orbital motion of light is needed to create its orbital speed centrifugal force equal to the force of an atomic attraction. This rate is equal to the atomic orbit well-known speed of light - (c). In aerodynamics this force is called the first space.

The atomic attraction is generated by the pressure gradient in the atomic vortex (uravn.4). The pressure gradient is the difference of pressure values in free ester stationary and the center of the vortex. In a free state, the pres sure is always constant and maximum. In the center of the vortex ether pressure depends on the rotational speed and air temperature. Upon heating of a substance (atom), the pressure $(\mathrm{P})$ atom in the center increases in proportion to the temperature $(\mathrm{T})$, in accordance with the law of Charles:

$$
\mathbf{T} \sim \mathbf{P}
$$


Then the pressure gradient must be reduced and, in accordance with equation (4) reduces the force of attraction of the vortex. In this case, there comes a predominance of centrifugal force $F_{c}$ over the forces of atomic, vortex attraction $F_{n}$. The speed of light is converted into the second cosmic speed. Light is removed from the atom at the most economical spiral trajectory according to calculations by Walter Hohmann. At the same time near the surface of the atom (at the lowest atomic orbits), the place flown away luminous flux takes a new ether flow. This stream is the same principle is converted into light. If the external effects (heating) is stored, the conversion and emission light from the atom will continue permanently. This is the nature of light. Light is generated and emitted not by the energy or mass of matter (atoms), but only by making the ether in the light under the influence of the atomic vortex gravitation and external influences (temperature). Light emission stop only when the external action (heating) or destruction of the atom, and with it the atomic vortex.

\section{Fig. 2 shows the movement of light aerodynamic configuration of the atom.}

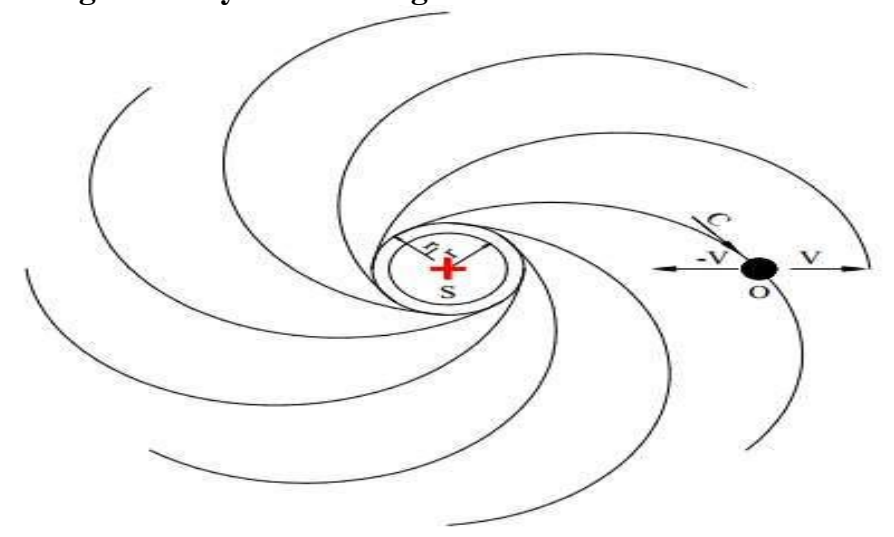

Fig. 1. S - light source (the atom), O - observer, $r$ - radius of the atom, $r_{1}$ - the radius of the orbit of the highest flux at his address in a calm state, prior to heating (at orbital velocity), $\mathrm{v}$ - relative velocity of removal or approximation between $\mathrm{S}$ and $\mathrm{O}, \mathrm{c}$ - velocity of light vector.

Fig. 2 shows: the observer $(\mathrm{O})$ to move relative to the light source $(\mathrm{S})$ with a speed v; light flux from the atom which trajectory intersects the observer $(\mathrm{O})$ at a large angle. Consequently, the speed of light relative to the observer $(\mathrm{O})$ is equal to the absolute

speed of light - c. Observer speed (v) with respect to the light source can not change the rate of arrival of light (c) to the observer.

Fig. 2 shows an aerodynamic scheme of movement of the light flux from a single atom. In the emission of light from the set of atoms aerodynamic design principle does not change, only multiplied many times. Light will always be supplied to each of the observer to any atom on the same helical path (at a large angle to the radius of the light field) with its constant velocity - v. Spiral light fluxes from these atoms can twist in different directions. The orbit planes of light fluxes arranged in space with any inclinations from each other. Thus, the light is almost infinite set of ultra-thin, light, spiral threads. These streams are combined into a single spherical light field. Radius and circumference of the light field in their values are directly proportional.

Consequently, the light field radially increases with the same speed of light.

If the viewer moves around the light source light over a circular orbital path, and in this case a considerable part of the light will make its spiral rotation along a plane perpendicular to the plane of movement of the observer. Consequently, this light will also enter the observer at a right angle with its constant velocity - v. Isotropy of light is also a consequence of the propagation of light streams through an infinite number of spiral trajectories.

Output. To put the speed of light (c) at the speed of anybody (v) in space is possible only according to the laws of vector algebra. Relative movement of any other body in space, light always moves at a right angle. Therefore, the relative velocity of the entering light to anybody will always be equal to the absolute speed of light, regardless of the speed of motion of the considered point $(\mathrm{O})$ relative to the light source $(\mathrm{S})$.

Mathematically this can be written:

$$
c^{-}+\bar{v}=c^{-}-\bar{v}=c^{-}
$$

\section{CONCLUSIONS}

The concept of the luminiferous ether was launched in the XVII century Rene Descartes. In the future, the wave theory, the luminiferous ether developed in the writings of Huygens. A detailed justification for this theory was in the XIX century in the framework of wave optics and electromagnetic theory of Maxwell. The proposed spiral model of light is very close to the concept of the wave motion of light and differs only in the trajectory of the light flux.

According to modern concepts light has a dual nature (wave-particle duality): - light has wave properties and is an electromagnetic wave, but is also the stream of particles - photons. It should be noted that until now scientists have not explored yet another important property of light: what is the mechanism and how can instantly disperse photons up to the maximum speed - c?! 
Spiral model of the origin and movement of light, this problem is not difficult, because the momentum of the movement all the magnetic flux received from the ether, which at the outbreak of the world itself was moving at a speed exceeding the speed of light.

Under Chapter 2 of this article can be argued that this is a consequence of the invariance of the light path of movement of the light flux. Researchers' relative velocity of light in their calculations are not stacked vector velocities and their modules. The movement of the luminous flux is not contrary to the laws of classical physics. Invariance was introduced into scientific consideration only because of a misunderstanding of the movement of light as straight and radial.

\section{REFERENCES}

[1].[1] The relative motion of the Earth and the Luminiferous ether. Albert A. Michelson, Master, U.S.Navy The American Journal of Science. 1881. III series. Vol XXII, No. 128. P. 120-129.

[2].Koninklijke Academie van Wetenschappen - te Amsterdam, 15, 1297 (1913)

[3].Einstein A. «Zur Elektrodynamik bewegter Korper» Ann Phys.- 1905.- Bd 17.- S. 891

[4].S. Orlov. Foundation of vortex gravitation, cosmology and cosmogony. Global journal of science Frontier research. Physic and Space Science Volume 12 issue 1 Version 1.0 January 2012

[5].Atsurovskiy. General ether-dynamics. Energoatomizdat. Moscow, Russia. 1990. Page 278. research. Physic and Space Science Volume 12 issue 1 Version 1.0 January 2012 